\title{
Research on the performance of T-type RHS Joints
}

\author{
Yan Liu* ${ }^{* 1, a}$, Fei Wang ${ }^{2, b}$, Qing Yang ${ }^{2, c}$, Xiaotong Shang ${ }^{2, d}$ \\ ${ }^{1}$ Shandong Provincal Key Laboratory of Civil Engineering Disaster Prevention and Mitigation, \\ Shandong University of Science and Technology, Qingdao, Shandong, 266590, China \\ ${ }^{2}$ College of Civil Engineering and Architecture, Shandong University of Science and Technology \\ Qingdao, Shandong 266590, China \\ adengpeng1226@163.com, b1003016081@qq.com ,'1364996979@qq.com, '173296033@qq.com
}

Keywords: inner stiffened plate, T-type RHS joints, seismic performance.

Abstract. The inner plate can be used to stiffen square tubular T-joint in order to improve the performance of square tubular T-joint and the radial stiffness of square tube. In this paper, tow methods of experiment and numerical simulation have been used to study the strengthening effect. Three scales of welded T-type RHS joints have been designed and three corresponding finite element modes have been established. Their anastomotic deformations verify the accuracy of the finite element model, boundary condition and loading mode by comparing the experiment and numerical simulation results. The research in the paper proved that RHS joints with inner stiffened plate had better ductility, energy dissipation and seismic performance because of the change of failure mode of specimen.

\section{Introduction}

The wall of the chord in the square tubular T-joint tends to plastic deformation because the branch members mainly bear axial loading [1]. Joint with Inner Stiffened Plate have been researched extensively all over the world because this joint not only can improve the radial stiffness of the chord and the ultimate capacity of the joint effectively, but also the stiffened plate located at the interior of the chord has no effect on the appearance of the structure. But researches of hysteretic performance of this joint under cyclic loading are limit [2-4]. The research by experiment and numerical simulation in the paper analyzed the effect of inner plate on the hysteretic behaviour of the joints and put forward the suggestion about the design of this type of joint.

\section{Contrast analysis of experiment and numerical stimulation of T-type RSH joints}

In this paper, quaci-static experiments on performance of 3 T-type RHS joints have been carried out. The size of main pipe of the specimens is $250 \mathrm{~mm} \times 250 \mathrm{~mm}$ whose thickness is $8 \mathrm{~mm}$, and the size of branch pipe is $150 \mathrm{~mm} \times 150 \mathrm{~mm}$ whose thickness is $6 \mathrm{~mm}$. Three specimens are with no stiffened plate, with stiffened plate of small hole and with stiffened plate of large hole respectively. All specimens are made of Q235B. The parameters of specimens are shown in Table 1 and Fig.1.

Table 1 Parameters of specimens (Unit: $\mathrm{mm}$ )

\begin{tabular}{|c|c|c|c|c|c|c|c|c|c|c|c|}
\hline Parameter & $l$ & $l_{0}$ & $d_{0}$ & $t_{0}$ & $l_{l}$ & $d_{1}$ & $t_{1}$ & $l_{0}$ & $d_{s}$ & $t_{s}$ & $\beta\left(d_{1} / d_{0}\right)$ \\
\hline T250-150 & 2500 & 2208 & 250 & 8 & 400 & 150 & 6 & - & - & - & 0.6 \\
\hline T250-150-S1 & 2500 & 2208 & 250 & 8 & 400 & 150 & 6 & 234 & 118 & 6 & 0.6 \\
\hline T250-150-S2 & 2500 & 2208 & 250 & 8 & 400 & 150 & 6 & 234 & 174 & 6 & 0.6 \\
\hline
\end{tabular}



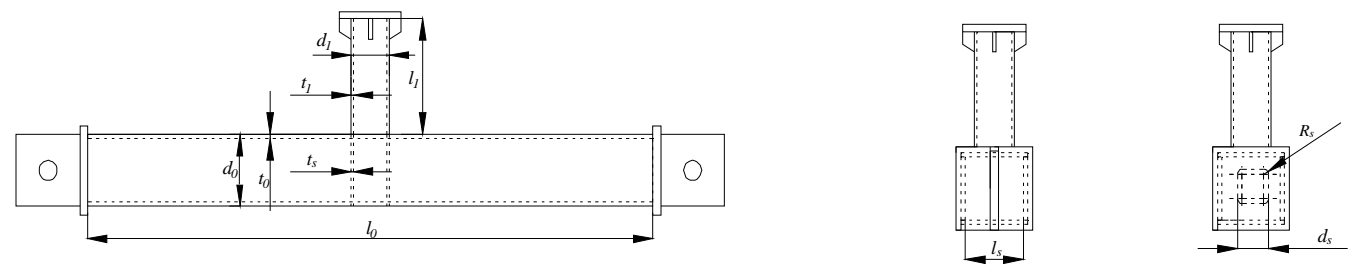

Fig.1 Geometric parameters of specimen

Analysis of T250-150.10kN is pre loaded before the experiment and then the load is controlled which is divided into five stages of $\pm 30 \mathrm{kN}, \pm 60 \mathrm{kN}, \pm 90 \mathrm{kN}, \pm 100 \mathrm{kN}$ and $\pm 110 \mathrm{kN}$. Each load control cycles one time, and when load control finished, the yield displacement $\Delta y$ is $14.202 \mathrm{~mm}$. Then the loading was controlled by the displacement. The yield displacement of numerical simulation of the component is $15.1 \mathrm{~mm}$, the yield load is $118 \mathrm{kN}$. The difference of the yield displacement between the experiment and simulation result is $5 \%$ and that of the yield load is $7.2 \%$.ANSYS numerical simulation is an ideal elastic-plastic constitutive relationship and the damage of the material is not considered, so the calculation error of the structure in the elastic stage is not obvious and the difference is less than $10 \%$, but the difference is gradually increasing in elastic-plastic stage.

As shown in Fig.2, the wall of the main pipe curves slightly when branch pipe is in compression in the second cycle of $1 \Delta y$, which is because radial load of main pipe transformed from axial load of branch pipe in compression makes the buckling of the pipe junction due to stress concentration and the bending moment at the web which is transformed from radial load of head of main pipe wall curve makes the web buckle.

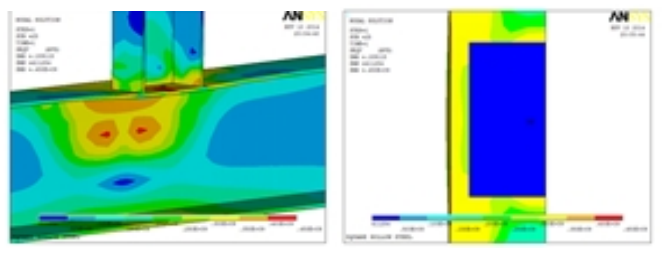

Fig. 2 Compression deformation of $1 \Delta y$

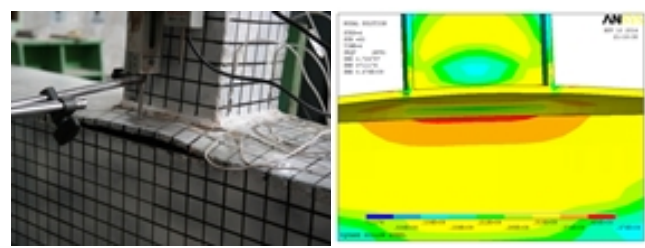

Fig.3 Destruction of joint

As shown in Fig. 3, the deformation of the top flange of the chord near the intersection between chord and branch increases when branch pipe is in tension in the first circulation of $2 \Delta y$ and the welds on the edge of the upper flange and web appear serious crack, then the experiment stops. It can clearly found from the numerical simulation that the stress concentration at the crack is obvious. For T250-150, AYSYS simulation results are in agreement with the results of experiment.

Analysis of T250-150-S1. The experiment procedure of T250-150-S1 is similar to T250-150. The load is divided into three stages of $\pm 76 \mathrm{kN}, \pm 152 \mathrm{kN}$ and $\pm 228 \mathrm{kN}$, and the yield displacement $\Delta \mathrm{y}$ is $17.932 \mathrm{~mm}$. Then the loading was controlled by the displacement. The yield displacement of the numerical simulation component is $16.4 \mathrm{~mm}$, and the yield load is $239 \mathrm{kN}$. The difference of the yield displacement between the experiment and the experiment results is $8 \%$ and the yield load difference is $1.3 \%$.

The whole bending of component is obvious when branch pipe is in compression in the first circulation of $2 \Delta y$. As shown in Fig.4, the upper flange appears concave obviously away from the weld of the joint about $50 \mathrm{~mm}$. The use of stiffened plate makes axial bearing capacity of branch pipe corresponding to main pipe increase greatly, which makes the yielding region transfer to outside of the joints. As shown in Fig. 5 and 6, when the branch pipe is in tension, upward deformation of main pipe and bucking on the side of chord between stiffening plates is obvious. The area on bottom of chord between stiffening plates is in depression obviously. Because the main pipe appears obvious deformation, the upper half part of the main pipe between the stiffened plates is in 
tension and other part is in compression. As the force increases to more than ultimate bearing capacity of main pipe, the main pipe appears local buckling.

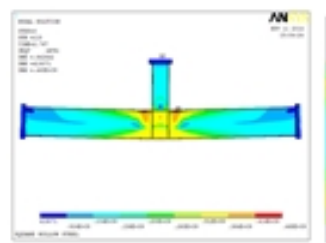

Fig.4 Compression deformation of $2 \Delta y$
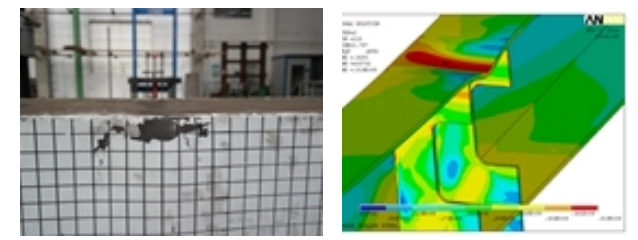

Fig.6 Depressed deformation on bottom of chord

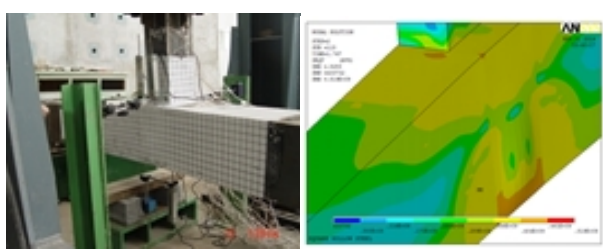

Fig.5 Bucking on the side of chord

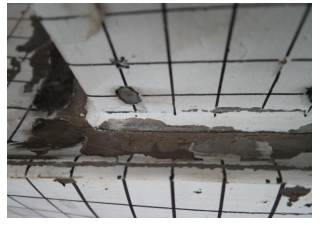

Fig.7 Destruction of weld on the edge of chord

As shown in Fig. 7, the welds near the intersection of pipes which are mainly concentrated in the length direction of the main pipe appear destruction when branch pipe is in tension in the circulation of $3 \Delta y$. When the brace is in compression, the local buckle of top flange of the chord is aggravated and the bearing capacity of the joint has reduced to $85 \%$ of the ultimate bearing capacity, which means the joint has been destroyed.

Analysis of T250-150-S2. The load is divided into three stages of $\pm 53 \mathrm{kN}, \pm 106 \mathrm{kN}$ and $\pm 109 \mathrm{kN}$. The yield displacement is $18.90 \mathrm{~mm}$, and then the loading was controlled by the displacement. The yield displacement of the numerical simulation component is $17.2 \mathrm{~mm}$ and the yield load is $172 \mathrm{kN}$. The difference of the yield displacement between the experiment and the simulation results is $8.9 \%$ and the difference of yield load is $8.2 \%$.

As shown in Fig.8, the whole downward deformation of component is obvious and local deformation of the joints is more serious when branch pipe is in compression in circulation of $1 \Delta y$. In contrast with the component without inner plates, the whole deformation of T250-150-S2 is more obvious and the local deformation is lighter, which is because the axial stiffness of stiffened joint is changed. In contrast with T250-150-S1, the whole deformation of T250-150-S2 is lighter and the local deformation is more serious, which is because the axial stiffness is less than T250-150-S1due to the larger hole size.
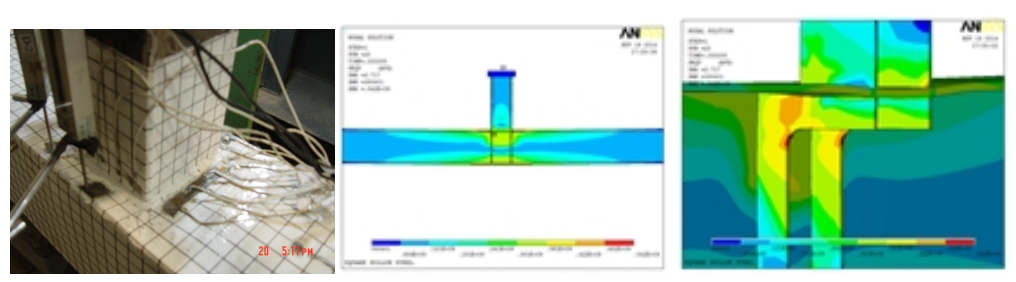

Fig. 8 Compression deformation of $1 \Delta \mathrm{y}$ circling
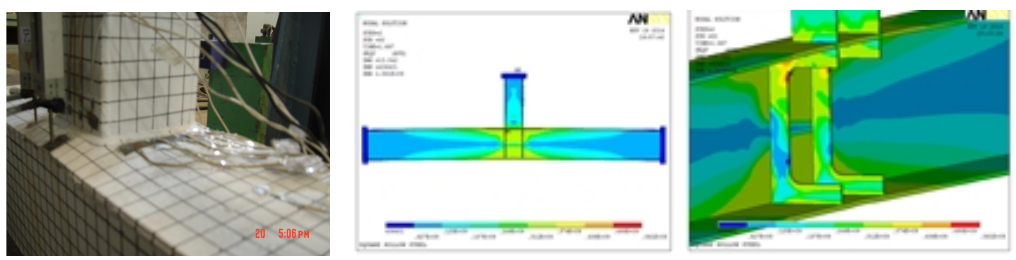

Fig.9 Tensile deformation of $1 \Delta y$ circling

As shown in Fig.9, upward bending of the whole component and a slight upward curve appears between the stiffened plates of bottom flange as branch pipe is in tension in circulation of $1 \Delta y$.The deformation of T250-150-S2 is smaller than that of T250-150-S1 and is larger than that of 
unstiffened joint, while local deformation of T250-150-S2 is more obvious than that of T250-150-S1and is less than that of unstiffened joint, which are because the stiffened plates changed the axial stiffness of the main pipe and the overall upward deformation of the component force the net distance between the stiffened plates to decrease.

As shown in Fig.10, when branch pipe is in compression in circulation of $2 \Delta y$, depressed deformation of chord on the intersection of pipes is obvious which is because the hole of stiffened plates are too large, and the overall deformation do not change significantly compared with in circulation of $1 \Delta y$. The downward bending of stiffened plates connected with the top flange increase. When the branch pipe is in tension, the top flange of main pipe buckles upward and the corner of welds on the intersection of pipes cracks slightly, which increases the overall deformation of the component.

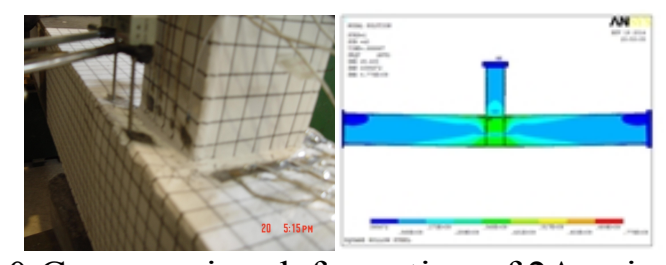

Fig. 10 Compression deformation of $2 \Delta y$ circling

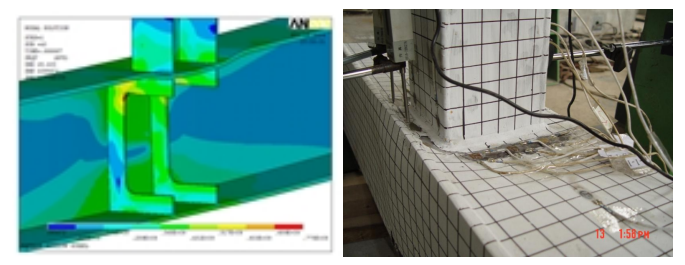

Fig.11 Destruction of the joint

As shown in Fig.11, the local buckling of top flange of chord on the intersection of pipes is serious and the middle and upper part of chord web curves obviously when branch pipe is in compression in circulation of $3 \Delta y$, which is because the pressure of branch pipe passed to the inner plates and inner plates bend. At this time, the bearing capacity of the joint is reduced to $85 \%$ of the ultimate bearing capacity and the joint is destroyed when the experiment is finished.

\section{Conclusion}

Through the comparison between the experiments and the numerical simulation, the results of ANSYS numerical simulation are in good agreement with the experimental results. The intersection of pipes of T-type RHS joint without stiffened plates tends to buckling easily because of the hollow main pipe. The joint with inner plates increases the axial stiffness of chord on the intersection of pipes and delays the local buckling of the intersection of pipes. The inner plates change the failure mode of T-type RHS joint which is overall bending and the ductility and energy dissipation ability has been improved which is more helpful to seismic resistance of structure. The hole size of the inner plates has great influence on the global and local deformation of T-type RHS joint under axial cyclic loading.

\section{Reference}

[1] Eastwood, W., Wood, A. A. Welded joints intubular structures involving rectangular resctions [J]. Univ. of Sheffileld, England, 1970: Section A Paper2

[2] Gibstein, M. B. The static strength of T-joints subjected to in-plane bending [J]. Det Norske Veritas Report, 1976: 76-137

[3] Sparrow K D, Stamenkovic A. Experimental Determination of the Ultimate Static Strength of T-joints in Circular Hollow Steel Section Subjects to Axial and Moment [J]. Proc.Int.Conf., Joints in Structural Steel Work, Teeside, 1981

[4] Wardenier J . Hollow section Joints [M]. Delft University Press, 1982 